\title{
How important is early magnetic resonance imaging of the temporomandibular joint for the treatment of children with juvenile idiopathic arthritis: a retrospective analysis
}

\author{
Raphael A Hauser ${ }^{1}$, Silke Schroeder ${ }^{1}$, Elvira Cannizzaro ${ }^{1}$, Lukas Muller², Christian J Kellenberger ${ }^{3}$ \\ and Rotraud K Saurenmann ${ }^{1,4^{*}}$
}

\begin{abstract}
Background: Temporomandibular joint (TMJ) arthritis is common in children with juvenile idiopathic arthritis (JIA), but often clinically asymptomatic. Magnetic resonance imaging (MRI) is the most reliable examination method, but requires sedation in young children. The aim of our study was to evaluate whether early TMJ MRI will change the treatment of patients with newly diagnosed JIA.

Methods: Single center chart review of all patients with a diagnosis of JIA between January 2007 and December 2010.

Results: We found 147 patients with newly diagnosed JIA during this period. In 111 (76\%) at least 1 MRI of the TMJ was available. Reasons why no TMJ MRI was done were parents' refusal (10), MRI of other locations (7), fixed dental appliances (16) and unclear cause (3). A diagnosis of TMJ arthritis based on increased joint enhancement on MRI was made in 91/111 (82\%) patients. The first MRI was done at a median interval of 5 months from the diagnosis of JIA, and $61 / 111$ patients (55\%) required sedation for their first MRI. TMJ arthritis was diagnosed in 53/61 (87\%) requiring sedation and in 34/50 (68\%) patients without sedation $(p=0.003)$. Following the first TMJ MRI, intra-articular steroid injections were performed into 107 TMJs of 60 patients. 48/147 (33\%) patients received at least one DMARD to control their disease, and in 9/48 (19\%) the first DMARD was started following the first TMJ MRI. Factors associated with TMJ involvement as demonstrated by MRI were JIA subtype $(p=0.007)$ and a younger age at diagnosis of JIA $(p=0.04)$.

Conclusion: In our cohort of newly diagnosed JIA patients TMJ arthritis was very common. Early TMJ MRI led to changes in treatment in $62 \%$ of patients with additional joint injections in 60 patients and start of systemic medication in 9 patients. We especially recommend performing TMJ MRI in young children even if they require sedation, as they have an increased rate of TMJ involvement.
\end{abstract}

Keywords: Juvenile idiopathic arthritis, Temporomandibular joint arthritis, Magnetic resonance imaging, Treatment

\section{Background}

Juvenile idiopathic arthritis (JIA) is the most common rheumatic disease in children under 16 years of age [1].

According to ILAR classification JIA is an arthritis of unknown etiology, with onset before the sixteenth birthday and a minimal duration of 6 weeks [2]. JIA is divided

\footnotetext{
* Correspondence: Traudel.Saurenmann@ksw.ch

'Rheumatology, University Children's Hospital, Steinwiesstrasse 75, 8032 Zurich, Switzerland

${ }^{4}$ Department of Pediatrics, Cantonal Hospital, Winterthur, Switzerland Full list of author information is available at the end of the article
}

into 7 subtypes based on clinical and laboratory parameters $[2,3]$. In all subtypes of JIA, one or both temporomandibular joints (TMJ) can be involved [4-6]. The reported frequency of TMJ involvement in the literature depends on the investigated population, the subtypes of JIA represented and the diagnostic methods used, and varies between $17 \%$ to $87 \%$ [4-10]. During the course of JIA, the TMJ may be the initial or even the only joint affected $[11,12]$. Often, however, the involvement of the TMJ occurs asymptomatically and without clinically detectable signs, for which reason the diagnosis is often

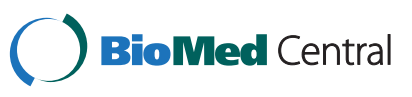


delayed [8,13-16]. Chronic inflammation of the TMJ can lead to severe mandibular growth disturbances [17-20]. Mandibular dysfunction and alterations in facioskeletal morphology such as micrognathia, retrognathism and mandibular asymmetry may be the consequences [21-25]. At the time when retrognathism or jaw asymmetry becomes obvious, the condyles are already irreversibly damaged [26,27]. An early diagnosis of TMJ arthritis is therefore essential to prevent such sequelae.

Previous studies have shown that magnetic resonance imaging (MRI) is the only method to diagnose TMJ arthritis at an early stage: Neither rheumatologic examination, nor orthodontic examination nor ultrasound (US) is able to reliably predict the presence or absence of MRI proven inflammation of the TMJ before structural changes have occurred $[7,8,16,28]$. But MRI is expensive, not always easily available and sedation is often needed in patients who cannot lie sufficiently still for the examination. The aim of our study was to evaluate how many patients with newly diagnosed JIA would profit from an early MRI for the further management of their arthritis.

\section{Methods}

\section{Patients}

The Pediatric Rheumatology database of our tertiary care pediatric referral center was searched for all patients with a diagnosis of JIA according to the ILAR 2001 criteria and a date of diagnosis between January 1st 2007 and December 31st 2010. Patients who had their permanent residence outside Switzerland were excluded.

The following data were then collected retrospectively from the patients' charts: Date of birth, sex, date of diagnosis of JIA, JIA subtype, status of antinuclear antibody (ANA) and rheumatoid factor (RF) testing and HLA B27 testing, date of diagnosis of uveitis, date of diagnosis of TMJ involvement, number and location of active joints at each clinic visit, type of medication received including start and stop date of treatments (if applicable), date, dose and location of intraarticular steroid injections performed, date and result of MRI of the TMJ performed. The study was approved by the Institutional and Governmental Ethics Review Board.

\section{Methods}

Definitions

For the diagnosis of JIA the ILAR Criteria, Edmonton Revision 2001 were used [2]. Diagnosis of TMJ involvement was exclusively based on the findings on MRI. Increased joint enhancement including the synovial membrane, joint space and/or the mandibular head was used to diagnose active TMJ arthritis and typical changes of the condylar head such as flattening, squaring and loss of height to diagnose TMJ deformation.

\section{MRI}

Both TMJs were imaged on a 1.5 Tesla system (Signa MR/i Twinspeed scanner, GE Medical Systems, Milwaukee, Wisconsin, USA) with a dedicated TMJ surface coil in closed-mouth position. Axial and coronal T2-weighted fast spin echo localizers were acquired for adjusting the subsequent sagittal oblique sequences perpendicular to each mandibular condyle and parallel to each mandibular ramus. Sagittal oblique images were acquired with a $2 \mathrm{~mm}$ slice thickness, without gap, $12 \mathrm{~cm}$ field of view and $256 \times 224$ matrix. First T1-weighted spoiled gradient echo images (flip angle $80^{\circ}$, TR $325 \mathrm{~ms}$, TE $4.2 \mathrm{~ms}$ ), proton density fast spin echo images (TR $2660 \mathrm{~ms}$, TE $25 \mathrm{~ms}$, ETL 8) and fatsaturated T2-weighted fast spin echo images (TR $2840 \mathrm{~ms}$, TE $86 \mathrm{~ms}$, ETL 16) were performed, followed by contrast-enhanced fat-saturated $\mathrm{T} 1$-weighted fast spin echo images (TR $600 \mathrm{~ms}$, TE $11 \mathrm{~ms}$, ETL 3) acquired within 5 minutes after intravenous administration of a single dose $(0.1 \mathrm{mmol} / \mathrm{kg}$ bodyweight $)$ of gadolinium based contrast medium (dimeglumine gadopentate, Magnevist, Bayer AG, Switzerland; or gadodiamide, Omniscan, GE Healthcare AG, Switzerland).

In patients who were unable to lie still for the duration of the examination the MRI was performed with propofol sedation according to hospital routine. This was usually the case for children under 6 years of age. In older children the indication for sedation was decided individually based on an informed discussion with the parents and the child. As of clinic routine the performance of an MRI of the TMJ joints was proposed to all patients/parents irrespective of clinical signs of TMJ involvement at the following time points during treatment of JIA:

- at diagnosis, if a patient presented with only a few active joints and was planned to receive intraarticular steroid injections only. In this case, all necessary intraarticular injections were conducted during the MRI sedation, if applicable. In patients who did not require sedation for the MRI, the TMJ MRI was performed before the joint injections were scheduled.

- after having achieved clinically inactive disease, if a patient was treated with systemic medication for polyarticular joint involvement

- in cases with a normal first TMJ MRI a follow-up MRI was proposed if clinical signs of TMJ arthritis appeared, if systemic medication was stopped, or 1-2 years after the first MRI.

\section{Statistical analysis}

Statistical analysis was performed using the JMP IN 8 software from the SAS Institute Inc, Cary NC, USA. Univariate analysis was performed using one way analysis 
of variance (ANOVA) or Chi square. Factors significantly associated with TMJ involvement were then tested in a multivariate stepwise regression and logistic regression model. A p value $\leq 0.05$ was considered a statistically significant difference.

\section{Results}

We found a total number of 147 patients with newly diagnosed JIA. In 111/147 patients (76\%) at least 1 MRI of the TMJ was available. Reasons why no TMJ MRI was done in the remaining 36 patients were parents' refusal (10), MRI of other locations in children requiring sedation (7) and fixed dental appliances (16), see Figure 1. In 3 patients the reason why no MRI had been performed remained unclear. For patients characteristics see Table 1.

The first MRI was done at a median interval of 5.04 (range 0-48.8) months from the diagnosis of JIA. In 29/ 111 (26\%) patients only one MRI was performed, 82/111 (74\%) patients had repeated MRI examinations. The maximum amount of MRI examinations performed in this cohort was 8 MRIs in one patient. The group of patients with and without MRI examination differed significantly in several aspects: Patients with MRI were significantly younger at diagnosis of JIA ( $p=0.0003$, ANOVA), ANA testing was more often positive $(p=0.04$, Chi Square), they had a significantly longer JIA duration ( $\mathrm{p}=0.0002$, ANOVA) and they were treated significantly more often with at least one DMARD ( $p=0.003$, Chi Square). In addition, there was a significant difference between the JIA subgroups receiving a MRI examination ( $p=0.01$, Chi Square): Patients with enthesitis related JIA $(10 / 19,53 \%)$ had the lowest rate of MRI examination, followed by patients with undifferentiated $(3 / 5,60 \%)$, oligoarticular $(49 / 70,70 \%)$, psoriatic $(7 / 8,88 \%)$, polyarticular $(26 / 28,93 \%)$, oligoarticular extended $(15 / 16$, $94 \%)$ and systemic $(1 / 1,100 \%)$ JIA.

A diagnosis of active TMJ arthritis was made in 91/ 111 (82\%) patients. This diagnosis was based on the first MRI in $87 / 111$ (78\%) patients. Patients with a diagnosis of TMJ arthritis were significantly younger at the time of JIA diagnosis than patients without a TMJ arthritis ( $\mathrm{p}=$ 0.04, ANOVA), they were more likely to have a positive ANA test $(15 / 20,75 \%), \quad(p=0.04$, Chi Square), more commonly had oligoarticular extended (15/91, 16\%), polyarticular (23/91, 25\%) JIA subtypes and significantly less common had enthesitis related JIA $(6 / 20,30 \%)(p=0.012$,

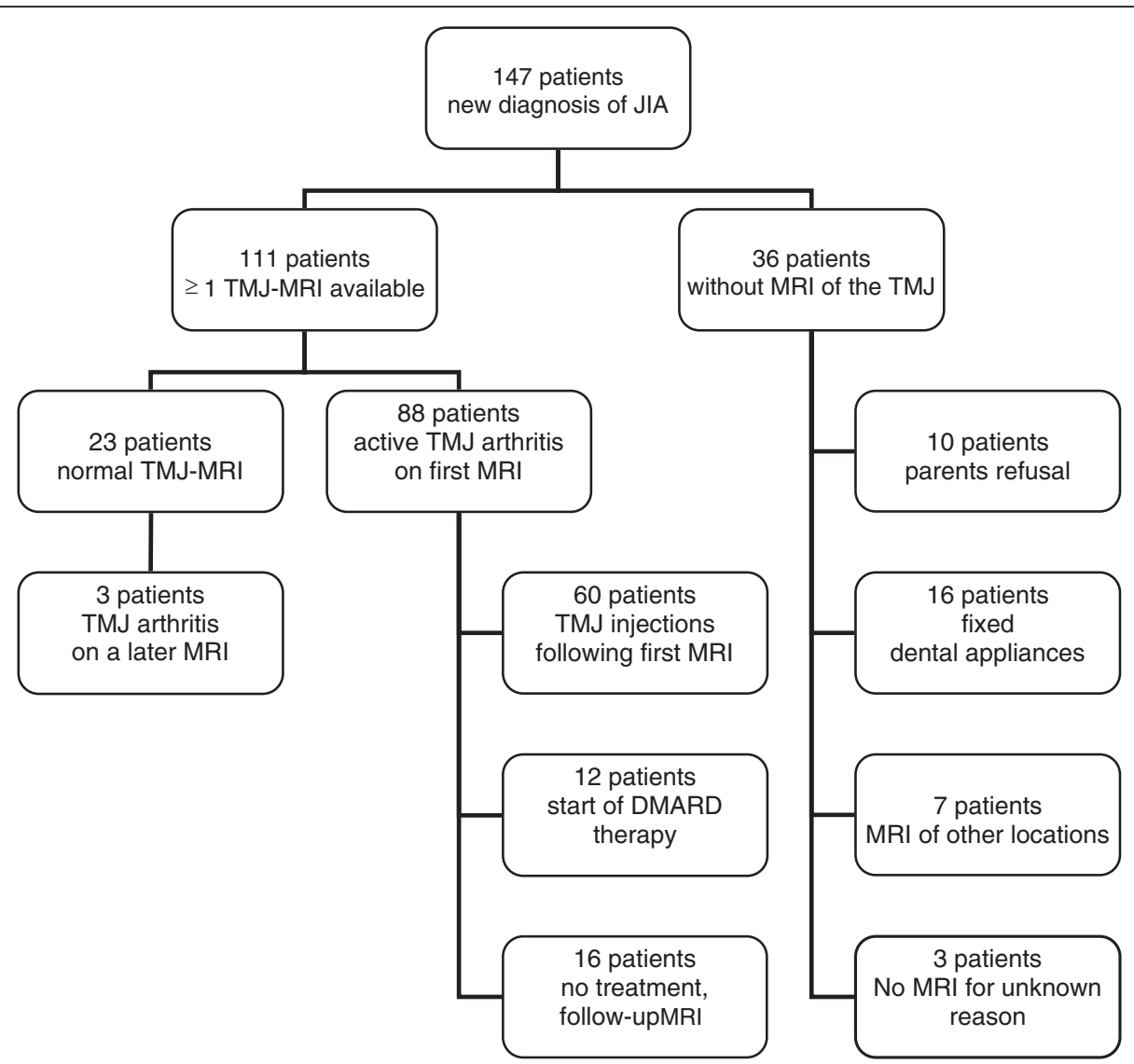

Figure 1 Flow sheet showing patients with TMJ MRI results and consequences on one arm and reasons why no MRI was performed on the other arm. 


\begin{tabular}{|c|c|c|c|c|c|}
\hline & \multirow[t]{2}{*}{ Total cohort } & \multicolumn{3}{|c|}{ MRI cohort } & \multirow{3}{*}{$\begin{array}{c}\text { Statistics } \\
\text { Comparison with/without } \\
\text { TMJ-Arthritis }\end{array}$} \\
\hline & & All & With TMJ-Arthritis & $\begin{array}{c}\text { Without } \\
\text { TMJ-Arthritis }\end{array}$ & \\
\hline & $n=147$ & $n=111$ & $\mathrm{n}=91$ & $n=20$ & \\
\hline Female (\% of cohort) & $88(60 \%)$ & $70(63 \%)$ & $60(66 \%)$ & $10(50 \%)$ & $p=0.2$ \\
\hline JIA subtypes (\% of cohort) & & & & & $p=0.007^{*}$ \\
\hline Enthesitis related & 19 (13\%) & $10(9 \%)$ & $4(4 \%)$ & $6(30 \%)$ & \\
\hline Oligoarticular & $70(48 \%)$ & 49 (44\%) & $40(44 \%)$ & $9(45 \%)$ & \\
\hline Extended oligoarticular & $16(11 \%)$ & $15(14 \%)$ & $15(16 \%)$ & 0 & \\
\hline Polyarticular RF neg & $28(19 \%)$ & $26(23 \%)$ & $23(25 \%)$ & $3(15 \%)$ & \\
\hline Psoriatic & $8(5 \%)$ & $7(6 \%)$ & $6(7 \%)$ & $1(5 \%)$ & \\
\hline Systemic & $1(0.7 \%)$ & $1(1 \%)$ & $1(1 \%)$ & 0 & \\
\hline Undifferentiated & $5(3 \%)$ & $3(3 \%)$ & $2(2 \%)$ & $1(5 \%)$ & \\
\hline ANA pos (\%) & $60(41 \%)$ & $51(46 \%)$ & $46(50 \%)$ & $5(25 \%)$ & $p=0.04^{*}$ \\
\hline RF pos (\%) & $1(0.7 \%)$ & $1(0.9 \%)$ & 0 & 1 & \\
\hline HLA B27 pos/tested (\%) & 18/124 (15\%) & $12 / 97(12 \%)$ & $8 / 78(10 \%)$ & $4 / 19(25 \%)$ & $p=0.2$ \\
\hline Uveitis (\% of cohort) & $7(5 \%)$ & $7(8 \%)$ & $6(7 \%)$ & $1(5 \%)$ & $p=0.7$ \\
\hline Age at JIA diagnosis in years (mean; median) & $7.3 ; 6.9$ & $6.6 ; 6.0$ & $6.3 ; 5.8$ & $8.2 ; 8.4$ & $p=0.04^{*}$ \\
\hline JIA disease duration in years (mean; median) & $2.0 ; 1.9$ & $2.2 ; 2.3$ & $2.3 ; 2.4$ & $1.6 ; 1.6$ & $p=0.01^{*}$ \\
\hline Age at TMJ diagnosis in years (mean; median) & & & $7.0 ; 6.4$ & & \\
\hline Time until TMJ diagnosis in years (mean; median) & & & $0.7 ; 0.4$ & & \\
\hline \multicolumn{6}{|l|}{ Medication } \\
\hline NSAID (\%) & $126(86 \%)$ & $96(86 \%)$ & 79 (86\%) & $17(85 \%)$ & \\
\hline Methotrexate (\%) & $47(32 \%)$ & $44(40 \%)$ & $37(41 \%)$ & $7(35 \%)$ & \\
\hline Etanercept (\%) & $14(10 \%)$ & $14(13 \%)$ & $14(15 \%)$ & 0 & \\
\hline Infliximab (\%) & $7(5 \%)$ & $6(5 \%)$ & $6(7 \%)$ & 0 & \\
\hline Prednisone (\%) & $11(7 \%)$ & $9(8 \%)$ & $8(9 \%)$ & $1(5 \%)$ & \\
\hline Sulfasalazine (\%) & $2(14 \%)$ & $1(1 \%)$ & 0 & $1(5 \%)$ & \\
\hline Leflunomide (\%) & $3(2 \%)$ & $2(2 \%)$ & $2(2 \%)$ & 0 & \\
\hline Adalimumab (\%) & $2(1 \%)$ & $2(2 \%)$ & $2(2 \%)$ & 0 & \\
\hline Tocilizumab (\%) & $1(0.5 \%)$ & $1(1 \%)$ & $1(1 \%)$ & 0 & \\
\hline At least 1 DMARD (\%) & $48(33 \%)$ & 45 (41\%) & $38(42 \%)$ & 7 (35\%) & \\
\hline
\end{tabular}

*statistically significant ( $p$-value $<0.05$ ).

Chi Square). Clinically, TMJ involvement was suspected already at the first clinical examination in 32/111 (29\%) patients and was confirmed by MRI in all of them. Sixteen of 21 patients $(76 \%)$ with $>3$ months of DMARD therapy at the moment of TMJ MRI had active TMJ arthritis, and in 28/111 (25\%) patients all other joints were clinically inactive at the time of the first MRI examination. A multivariate stepwise regression analysis was performed with all factors significantly associated with TMJ arthritis in the univariate analysis: The only factor with statistically significant negative association with TMJ involvement was the JIA subtype enthesitis related arthritis $(\mathrm{p}=0.002)$.

Sixty one of 111 patients (55\%) required sedation for their first MRI. TMJ arthritis was diagnosed in 53/61
(87\%) patients with sedation in the first MRI and there was a significantly greater chance for diagnosis of TMJ arthritis in patients requiring sedation for the MRI examination ( $\mathrm{p}=0.003$, Chi Square). Of the 61 patients with sedation, $44(72 \%)$ received an injection in one (10 patients) or both (34 patients) TMJs or into other joints (38 patients) during the same sedation. In another 3/61 (5\%) a DMARD therapy was started following the first MRI.

Thirty four of $50(68 \%)$ patients without sedation for their first MRI examination were diagnosed with TMJ arthritis. As a result, a DMARD therapy was started in $6 / 50(12 \%)$ patients, and 16/50(32\%) patients received an injection in one (3 patients) or both (13 patients) TMJs 
within the following 2 months. Overall, early TMJ MRI led to changes in treatment in $69 / 111(62 \%)$ patients.

\section{Discussion}

In this study we examined the influence of TMJ MRI on the therapeutic management of an inception cohort of 147 patients with newly diagnosed JIA. TMJ MRI was performed in 111 patients at a time point where the result would influence treatment decisions. Early TMJ MRI led to a diagnosis of TMJ arthritis in $78 \%$ and changes in treatment in $62 \%$ of our patients, with additional joint injections in 60 patients and systemic medication started in 9 patients. The rate of treatment changes was even higher (72\%) in the very young children who required sedation for the MRI. To our knowledge, this is the first study examining this correlation.

Of special interest is the even higher rate of additional joint injections in patients who required sedation for the MRI. Many parents may be reluctant to have a MRI performed, especially if their child has no symptoms related to a possible TMJ involvement and would need a sedation to undergo the procedure. In this situation it is helpful for the treating physician to provide evidence based reasons to convince the parents about the necessity of this further examination for the optimal treatment of their child. Our result is in keeping with previous reports of a higher frequency of TMJ arthritis in patients with earlier disease onset: Twilt et al. [5] found TMJ arthritis in $45 \%$ of 97 patients with JIA, the onset of the disease was at an average of 1 year and 7 months earlier compared to JIA without TMJ. Cannizzaro et al. [9] found TMJ arthritis in 39\% of 223 patients with JIA, and TMJ involvement was significantly associated with a younger age at onset of JIA.

TMJ arthritis based on the first MRI examination was very common in our cohort with a frequency of $78 \%$. Other studies with TMJ MRI early after JIA diagnosis found similar frequencies of active TMJ arthritis: Weiss et al. [8] examined 32 patients within 8 weeks after JIA diagnosis and 75\% had TMJ arthritis. Küseler et al. [7] examined 15 newly diagnosed JIA patients and $87 \%$ had TMJ arthritis, and Müller et al. [28] examined 30 patients and 63\% had TMJ involvement.

In relation to the prevalence of TMJ arthritis in the literature, which varies between 17 and 87\% [4-10], our result is located at the upper edge. According to Ringold et al. [29], the large variation is attributed primarily to differences in inclusion criteria and diagnostic methods used. The later is of special importance at the onset of TMJ arthritis, because contrast-enhanced MRI is able to show early inflammatory changes, whereas other methods such as orthopantomogram (OPT), computed tomography $(\mathrm{CT})$ or ultrasound are unable to detect TMJ arthritis before mandibular head deformity has occurred $[7,8,28,30]$.

Interestingly, most long-term studies, including those using MRI as a diagnostic method, will find rates of TMJ pathology of around 30-45\% [5,9,31,32]: Stoll et al. [31] found signs of acute or chronic TMJ arthritis after a mean disease duration of 2.5 years in contrast-enhanced MRI in only $43 \%$ of 185 patients and Argyropoulou et al. [32] found abnormal condyles after a mean disease duration of 7.3 years in $32 \%$ of 46 JIA patients with TMJ MRI and enhanced pannus in $45 \%$.

Firstly, these findings raise the question, whether TMJ inflammation might be more active at the onset of JIA. Pedersen et al. [30] performed repeated TMJ MRI in 15 JIA patients prospectively 4 times within 2 years and found that nearly all had inflammatory signs at some point in time. Interestingly, on long-term follow-up improvement as well as worsening of the condylar shape have been demonstrated [33,34]. Why a presumably undulating inflammatory process will lead to progressive destruction in some patients but allows condylar remodelling in others remains unclear so far. Based on the hypothesis that the amount of inflammation present over a certain period of time correlates with the accrued damage, early therapeutic intervention would be the most adequate answer to this unpredictable disease course.

Another question would be, whether TMJ arthritis is overdiagnosed in MRI. Tzaribachev et al. [35] found that the majority (94\%) of non-rheumatic children had no MRI abnormalities in TMJ MRI. Also, Küseler et al. [36] found a high accordance of contrast enhancement on MRI with histopathologic findings of inflammation. Also Kottke et al. [37] found no pathologic contrast enhancement in 27 children undergoing head MRI without underlying rheumatic disease. In contrast, von Kalle et al. [38] examined 46 children with head-MRI for reasons other than TMJ disease and found contrastenhancement of the soft joint tissue and the condyles in all of them.

In only $76 \%$ of our patients a MRI examination was performed. The main reason why no MRI was performed was fixed dental appliances in teenage patients. While this explains why so many patients with enthesitis related JIA (ERA) had no TMJ MRI performed, it also leaves us with the question whether undiagnosed TMJ arthritis may in some cases have contributed to the need for orthodontic treatment in those teenage patients? On the other hand, these patients certainly had thorough examination including imaging as part of their orthodontic treatment and it is therefore unlikely that major changes of the condylar head should have escaped the attention of the treating physician. Another important reason for parents to refuse MRI examination was the need for sedation in asymptomatic children. In our 
cohort 55\% required sedation for their first MRI, $87 \%$ out of them had TMJ arthritis. Interestingly, there was a significantly greater chance for diagnosis of TMJ arthritis in patients who required sedation for the MRI examination. This raises the question whether the sedation itself may have influenced the result by providing a better examination quality. However, the association of younger age at diagnosis with an increased risk for TMJ involvement has been long recognized in studies using different imaging methods for the diagnosis $[5,9]$.

Our study is limited by its retrospective nature and by a relatively high number of patients who never had a MRI examination of the TMJs. Especially for the ERA subgroup our results are not conclusive for reasons mentioned previously. Although we routinely propose a TMJ MRI to our patients, we cannot completely exclude a selection bias favouring patients with longer disease duration, JIA diagnosis at a young age and with clinically more severe JIA subtypes. The true rate of TMJ involvement may therefore be lower than our results suggest.

\section{Conclusions}

Based on the early staged MRI, we were able to diagnose a significant amount of clinically asymptomatic children with TMJ arthritis and were able to adapt the treatment with the introduction of systemic medication and/or TMJ injections at an early stage of the disease. In conclusion, we recommend performing MRI of TMJ at an early stage of JIA diagnosis, especially in young children and even if sedation is required.

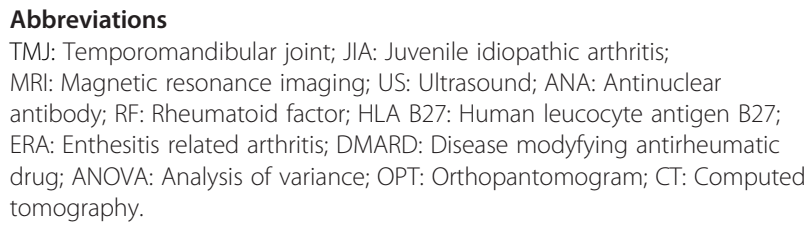

\section{Competing interests}

The authors declare that they have no competing interests.

\section{Authors' contributions}

$\mathrm{RS}, \mathrm{CK}, \mathrm{LM}$ study design. RH, SS, EC and RS data collection, $\mathrm{RH}$ and RS statistical analysis, interpretation of the data, manuscript preparation. CK, LM critical review of the manuscript. All authors read and approved the final manuscript.

\section{Acknowledgements}

We thank Fiona Possa-Copeland and Caroline Oechsle for their helpfulness throughout the time of the development of this article.

\section{Author details}

${ }^{1}$ Rheumatology, University Children's Hospital, Steinwiesstrasse 75, 8032 Zurich, Switzerland. ${ }^{2}$ Orthodontics and Pediatric Dentistry, University of Zurich, Zurich, Switzerland. ${ }^{3}$ Diagnostic Imaging, University Children's Hospital, Steinwiesstrasse 75, 8032 Zurich, Switzerland. ${ }^{4}$ Department of Pediatrics, Cantonal Hospital, Winterthur, Switzerland.

Received: 3 March 2014 Accepted: 8 July 2014

Published: 30 August 2014

\section{References}

1. Gäre BA: Epidemiology of rheumatic disease in children. Curr Opin Rheumatol 1996, 8:449-454

2. Petty RE, Southwood TR, Manners P, Baum J, Glass DN, Goldenberg J, He X Maldonado-Cocco J, Orozco-Alcala J, Prieur AM, Suarez-Almazor ME, Woo P: International League of Associations for Rheumatology classification of juvenile idiopathic arthritis: second revision, Edmonton, 2001. J Rheumatol 2004, 31:390-392.

3. Minden K, Niewerth M: Juvenile idiopathic arthritis-clinical subgroups and classification. Z Rheumatol 2008, 67:100, 102-106, 108-110.

4. Pedersen TK, Jensen JJ, Melsen B, Herlin T: Resorption of the temporomandibular condylar bone according to subtypes of juvenile chronic arthritis. J Rheumatol 2001, 28:2109-2115.

5. Twilt M, Mobers SM, Arends LR, ten Cate R, van Suijlekom-Smit L: Temporomandibular involvement in juvenile idiopathic arthritis. J Rheumatol 2004, 31:1418-1422.

6. Billiau AD, Hu Y, Verdonck A, Carels C, Wouters C: Temporomandibular joint arthritis in juvenile idiopathic arthritis: prevalence, clinical and radiological signs, and relation to dentofacial morphology. J Rheumatol 2007, 34:1925-1933.

7. Küseler A, Pedersen TK, Herlin T, Gelineck J: Contrast enhanced magnetic resonance imaging as a method to diagnose early inflammatory changes in the temporomandibular joint in children with juvenile chronic arthritis. J Rheumatol 1998, 25:1406-1412.

8. Weiss PF, Arabshahi B, Johnson A, Bilaniuk LT, Zarnow D, Cahill AM, Feudtner C, Cron RQ: High prevalence of temporomandibular joint arthritis at disease onset in children with juvenile idiopathic arthritis, as detected by magnetic resonance imaging but not by ultrasound. Arthritis Rheum 2008, 58:1189-1196.

9. Cannizzaro E, Schroeder S, Müller LM, Kellenberger CJ, Saurenmann RK Temporomandibular joint involvement in children with juvenile idiopathic arthritis. J Rheumatol 2011, 38:510-515.

10. Mayne JG, Hatch GS: Arthritis of the temporomandibular joint. J Am Dent Assoc 1969, 79:125-130.

11. Martini G, Bacciliero U, Tregnaghi A, Montesco MC, Zulian F: Isolated temporomandibular synovitis as unique presentation of juvenile idiopathic arthritis. J Rheumatol 2001, 28:1689-1692.

12. Scolozzi $P$, Bosson $G$, Jaques $B$ : Severe isolated temporomandibular joint involvement in juvenile idiopathic arthritis. J Oral Maxillofac Surg 2005, 63:1368-1371.

13. Svensson B, Adell R, Kopp S: Temporomandibular disorders in juvenile chronic arthritis patients. A clinical study. Swed Dent J 2000, 24:83-92.

14. Tzaribachev N, Benseler SM, Tyrrell PN, Meyer A, Kuemmerle-Deschner JB: Predictors of delayed referral to a pediatric rheumatology center. Arthritis Rheum 2009, 61:1367-1372.

15. Larheim TA, Höyeraal HM, Stabrun AE, Haanaes HR: The temporomandibular joint in juvenile rheumatoid arthritis. Radiographic changes related to clinical and laboratory parameters in 100 children. Scand J Rheumatol 1982, 11:5-12.

16. Arabshahi B, Cron RQ: Temporomandibular joint arthritis in juvenile idiopathic arthritis: the forgotten joint. Curr Opin Rheumatol 2006, 18:490-495.

17. Ronchezel MV, Hilário MO, Goldenberg J, Lederman HM, Faltin K, de Azevedo MF, Naspitz CK: Temporomandibular joint and mandibular growth alterations in patients with juvenile rheumatoid arthritis. J Rheumatol 1995, 22:1956-1961.

18. Kjellberg H: Juvenile chronic arthritis. Dentofacial morphology, growth, mandibular function and orthodontic treatment. Swed Dent J Supp/ 1995 109:1-56.

19. Twilt M, van der Giesen E, Mobers SM, ten Cate R, van Suijlekom-Smit LW: Abrupt condylar destruction of the mandibula in juvenile idiopathic arthritis. Ann Rheum Dis 2003, 62:366-367.

20. Huntjens E, Kiss G, Wouters C, Carels C: Condylar asymmetry in children with juvenile idiopathic arthritis assessed by cone-beam computed tomography. Eur J Orthod 2008, 30:545-551.

21. Twilt M, Schulten AJ, Nicolaas P, Dülger A, van Suijlekom-Smit LW: Facioskeletal changes in children with juvenile idiopathic arthritis. Ann Rheum Dis 2006, 65:823-825.

22. Kjellberg H, Fasth A, Kiliaridis S, Wenneberg B, Thilander B: Craniofacial structure in children with juvenile chronic arthritis (JCA) compared with healthy children with ideal or postnormal occlusion. Am J Orthod Dentofacial Orthop 1995, 107:67-78. 
23. Kjellberg H: Craniofacial growth in juvenile chronic arthritis. Acta Odontol Scand 1998, 56:360-365.

24. Sidiropoulou-Chatzigianni S, Papadopoulos MA, Kolokithas G: Dentoskeletal morphology in children with juvenile idiopathic arthritis compared with healthy children. J Orthod 2001, 28:53-58.

25. Twilt M, Schulten AJ, Prahl-Andersen B, van Suijlekom-Smit LW: Long-term follow-up of craniofacial alterations in juvenile idiopathic arthritis. Angle Orthod 2009, 79:1057-1062.

26. Svensson B, Larsson A, Adell R: The mandibular condyle in juvenile chronic arthritis patients with mandibular hypoplasia: a clinical and histological study. Int J Oral Maxillofac Surg 2001, 30:300-305.

27. Sidiropoulou-Chatzigianni S, Papadopoulos MA, Kolokithas G: Mandibular condyle lesions in children with juvenile idiopathic arthritis. Cleft Palate Craniofac J 2008, 45:57-62.

28. Müller L, Kellenberger CJ, Cannizzaro E, Ettlin D, Schraner T, Bolt IB, Peltomäki T, Saurenmann RK: Early diagnosis of temporomandibular joint involvement in juvenile idiopathic arthritis: a pilot study comparing clinical examination and ultrasound to magnetic resonance imaging. Rheumatology (Oxford) 2009, 48:680-685.

29. Ringold S, Cron RQ: The temporomandibular joint in juvenile idiopathic arthritis: frequently used and frequently arthritic. Pediatr Rheumatol Online J 2009, 7:11.

30. Pedersen TK, Küseler A, Gelineck J, Herlin T: A prospective study of magnetic resonance and radiographic imaging in relation to symptoms and clinical findings of the temporomandibular joint in children with juvenile idiopathic arthritis. J Rheumatol 2008, 35:1668-1675.

31. Stoll ML, Sharpe T, Beukelman T, Good J, Young D, Cron RQ: Risk factors for temporomandibular joint arthritis in children with juvenile idiopathic arthritis. J Rheumatol 2012, 39:1880-1887.

32. Argyropoulou Ml, Margariti PN, Karali A, Astrakas L, Alfandaki S, Kosta P, Siamopoulou A: Temporomandibular joint involvement in juvenile idiopathic arthritis: clinical predictors of magnetic resonance imaging signs. Eur Radiol 2009, 19:693-700.

33. Arvidsson LZ, Flatø B, Larheim TA: Radiographic TMJ abnormalities in patients with juvenile idiopathic arthritis followed for 27 years. Oral Surg Oral Med Oral Pathol Oral Radiol Endod 2009, 108:114-123.

34. Twilt M, Schulten AJ, Verschure F, Wisse L, Prahl-Andersen B, van Suijlekom-Smit LW: Long-term followup of temporomandibular joint involvement in juvenile idiopathic arthritis. Arthritis Rheum 2008, 59:546-552.

35. Tzaribachev N, Fritz J, Horger M: Spectrum of magnetic resonance imaging appearances of juvenile temporomandibular joints (TMJ) in non-rheumatic children. Acta Radiol 2009, 50:1182-1186.

36. Küseler A, Pedersen TK, Barlach J, Gelineck J, Sangill R, Melsen B, Herlin T: Contrast-enhanced MRI compared to histological findings in the temporomandibular joint of antigen-induced arthritis in young rabbits. Clin Exp Rheumatol 2004, 22:441-446.

37. Kottke R, Saurenmann T, Grotzer M, Kellenberger C: MRI of paediatric temporomandibular joints - normal findings [abstract]. Pediatr Radiol 2008, 38(Suppl. 3):S535-S536.

38. von Kalle T, Winkler P, Stuber T: Contrast-enhanced MRI of normal temporomandibular joints in children-is there enhancement or not? Rheumatology (Oxford) 2013, 52:363-367 (Epub 2012 Oct 11).

\section{doi:10.1186/1546-0096-12-36}

Cite this article as: Hauser et al.: How important is early magnetic resonance imaging of the temporomandibular joint for the treatment of children with juvenile idiopathic arthritis: a retrospective analysis. Pediatric Rheumatology 2014 12:36.

\section{Submit your next manuscript to BioMed Central and take full advantage of:}

- Convenient online submission

- Thorough peer review

- No space constraints or color figure charges

- Immediate publication on acceptance

- Inclusion in PubMed, CAS, Scopus and Google Scholar

- Research which is freely available for redistribution

Submit your manuscript at www.biomedcentral.com/submit
C Biomed Central 